\title{
A Project to Design and Build the Magnets for a New Test Beamline, the ATF2, at KEK
}

\author{
Cherrill M. Spencer, Ryuhei Sugahara, Mika Masuzawa, Benoit Bolzon, Andrea Jeremie
}

\begin{abstract}
In order to achieve the high luminosity required at the proposed International Linear Collider (ILC), it is critical to focus the beams to nanometer size with the ILC Beam Delivery System, and to maintain the beams' collisions with a nanometerscale stability. To establish the technologies associated with this ultra-high precision beam handling, a special beamline has been designed and built as an extension of the existing extraction beamline of the Accelerator Test Facility at KEK, Japan. The ATF provides an adequate ultra-low emittance electron beam that is comparable to the ILC requirements; the ATF2 mimics the ILC final focus system to create a tightly focused, stable beam. There are 37 magnets in the ATF2, 29 quadrupoles, 5 sextupoles and 3 bends. These magnets had to be acquired in a short time and at minimum cost, which led to various acquisition strategies; but nevertheless they had to meet strict requirements on integrated strength, physical dimensions, compatibility with existing magnet movers and beam position monitors, mechanical stability and field stability and quality. This paper will describe how 2 styles of quadrupoles, 2 styles of sextupoles, one dipole style and their supports were designed, fabricated, refurbished or modified, measured and aligned by a small team of engineers from 3 continents.
\end{abstract}

Index Terms-Accelerator magnets, magnetic measurements, room temperature magnets.

\section{INTRODUCTION}

A $\mathrm{n}$ important technical challenge of the proposed International Linear Collider is the collision of extremely small beams of a few nanometers $(\mathrm{nm})$ in size. This challenge has three distinct issues: creating small emittance beams, preserving the emittance during acceleration and transport, focusing the beams to a few nanometers and colliding them. The Accelerator Test Facility (ATF) at KEK was built to create small emittance beams, and has succeeded in obtaining an un-normalized vertical emittance of 10 picometers. picoradians, that is comparable to the ILC requirements.

The ATF2 facility, which uses the beam extracted from the ATF damping ring, was constructed to address two major

\footnotetext{
Manuscript received 20 October 2009. (This work was supported by Department of Energy (USA) contract DE-AC02-76SF00515; Agence Nationale de la Recherche (France) contract ANR-06-BLAN-0027).

Cherrill M. Spencer is with the SLAC National Accelerator Laboratory, Menlo Park, CA 94205, USA (phone: 650-926-3474; fax: 650-926-2407; email: cherrill@slac.stanford.edu).

Ryuhei Sugahara and Mika Masuzawa are with High Energy Accelerator Research Organization (KEK), Tsukuba, Ibaraki, Japan (e-mail: sugahara@post.kek.jp, masuzawa@post.kek.jp)

Benoit Bolzon and Andrea Jeremie are with LAPP, Annecy-le-Vieux, France (e-mail: bolzon@lapp.in2p3.fr, Andrea.Jeremie@lapp.in2p3.fr).
}

challenges of ILC: focusing the $1.3 \mathrm{GeV}$ beams to $37 \mathrm{~nm}$ using an ILC-like final focus and providing nanometer stability [1].

This paper describes the international effort to design, build, support and align the 37 magnets in ATF2's final focus. They were specified by a lattice designed initially in 2004; it evolved for 2 years and was finalized in 2006. Fig. 1 shows the positions of all components in the last $30 \mathrm{~m}$ of the ATF2 beamline's final focus (FF) section.

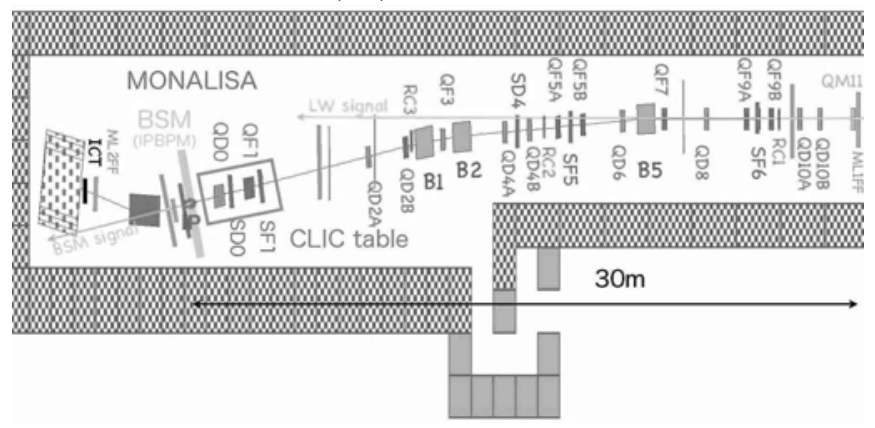

Fig. 1 Schematic of magnets and diagnostic instruments in ATF2 final focus

\section{OVERVIEW OF MAGNET PROCUREMENT}

The procurement of the new magnets started in January 2005. The various ways they were procured are described below. Installation of all magnets was finished in November 2008 and beam was first run through the beamline in December 2008. Commissioning is ongoing.

The required integrated magnet strengths in all 37 magnets were easy to achieve with water-cooled room temperature magnets. Engineering issues and challenges emanated from the stringent field quality requirements and the required positional stability of the magnets; coupled with the desire to spend as little money as possible.

\section{A. Philosophy \& Constraints of Magnet Procurement}

In general we took steps to minimize the cost of the new magnets and to produce them in timely way; we did this by these strategies:

1. Using existing magnets from SLAC and KEK when appropriate

2. Modifying existing magnets

3. Modifying existing magnet designs to save on engineering and drawing time

4. Using existing magnet movers

5. Using existing adjustable mounts

There were constraints on magnet sizes, apertures, coil ends, operating currents and voltages, because they had to fit in with the existing movers and the beam height from the floor of $1.2 \mathrm{~m}$; they had to interface with 2 different styles of Beam 
Position Monitors (BPM), and they had to fit in with a new, high reliability power supply which had current and voltage limits of 200 amps and 30 volts.

\section{B. Field Quality, Vibrational and Alignment Tolerances}

In order to achieve the $37 \mathrm{~nm}$ spot size the beam's emittance must not increase above $12 \mathrm{pm} \cdot \mathrm{prad}$ as it passes through the beamline. Higher multipoles in the magnets, magnets vibrating between 0.1 and $100 \mathrm{~Hz}$ and mis-aligned magnets will all cause the beam size to blow up. So the beam physicists set tight tolerances on these parameters, which vary from magnet to magnet, here are some sample tolerances. The sextupole/quadrupole at $1 \mathrm{~cm}$ tolerance in the $\mathrm{FF}$ quads ranges from $0.04 \%$ to $38 \%$. The sextupole/quad tolerance on QF1 and QD0 is very tight, particularly at QF1 where the horizontal beamsize is large $(3.5 \mathrm{~mm}),<0.03 \%$ at $1 \mathrm{~cm}$ was negotiated with the beam physicist, because the original tolerance of $0.0053 \%$ on QD0 was too small to even be measurable.

For the FF quadrupoles and sextupoles the vibrational tolerances range from 0.1 to $1 \mu \mathrm{m}$; the last 2 quads' vibrational tolerances are described in section IV. Without additional tuning, magnet alignment tolerances with respect to the design lattice have been calculated to be $1-5 \mu \mathrm{m}$, this is not achievable. With tuning procedures it has been shown that alignment tolerances of $300 \mu \mathrm{m}$ can be tolerated.

\section{Accessories to the Magnets}

The ATF2 beamline, in addition to the final focus, includes a diagnostics section to make measurements of the beam shape and correct it at the entrance to the final focus. In order to measure the beam spot size an interferometer-based beam size monitor (BSM, also called Shintake Monitor [2]) is used. To measure the beam orbit and maintain the beam size with feedback, the beamline magnets are equipped with sub-micron resolution cavity-BPMs and are placed on 3-axis remotely controlled mechanical movers. The latter had been designed and made for the Final Focus Test Beam (FFTB) at SLAC.

\section{DETAILS OF EACH STYLE OF MAGNET PROCURED}

The main parameters of the 5 styles of magnets are listed in Table I; each style is described in detail below.

TABLE I. Summary of Magnet Style Specifications

\begin{tabular}{lcccc}
\hline \hline $\begin{array}{c}\text { Magnet } \\
\text { Style }\end{array}$ & $\begin{array}{c}\text { Aperture } \\
\text { (gap or } \\
\text { diameter) } \\
\mathrm{mm}\end{array}$ & $\begin{array}{c}\text { Steel } \\
\text { Core } \\
\text { length } \\
\mathrm{mm}\end{array}$ & $\begin{array}{c}\text { Integrated } \\
\text { strength } \\
\text { range, T or } \\
\mathrm{T} \cdot \mathrm{m} \text { or T/m }\end{array}$ & $\begin{array}{c}\text { Current } \\
\text { for max } \\
\text { strength } \\
\mathrm{A}\end{array}$ \\
\hline FF quad & 32 & 180 & $2.46-10.88 \mathrm{~T}$ & 136.2 \\
FF dipole & 38 & 575 & $0.131-0.223 \mathrm{~T} \cdot \mathrm{m}$ & 163.6 \\
FF sext. & 41.28 & 89.66 & $3.43-64.66 \mathrm{~T} / \mathrm{m}$ & 28.5 \\
FD quad & 50 & 450 & $3.2-5.86 \mathrm{~T}$ & 132.2 \\
FD sext. & 54 & 76.2 & $11.5-19.37 \mathrm{~T} / \mathrm{m}$ & 6.5 \\
\hline \hline
\end{tabular}

\section{A. $27 \mathrm{FF}$ and extraction line quads, "QEA":}

Upon consideration of the strength and aperture requirements of the 14 final focus quads, 6 matching section quads and 7 extraction quads, we decided to make them all the same design; it was based on an existing KEK solid steel core, water-cooled coils quad design, which was modified for ATF2 use. The Institute of High Energy Physics (IHEP), Beijing made a complete drawing set according to our detailed requirements and standards. 28 quads (one spare) were fabricated at the IHEP shops, measured at IHEP and again at KEK. The KEK measurements are reported at this conference [3]. This was a novel situation for the authors: a magnet designed in the USA, to be made in China and operated in Japan, so we had issues of materials, e.g. availability of copper conductor; fittings thread sizes; standards for operating coils, each country's " usual practices" were different from the other countries'. Discussions took place (sometimes lengthy) to resolve which practice to use. This is a good example of the issues that will arise when designing and making the $~ 13,000$ ILC magnets all over the world.

Fig.2 is a photo of part of the ATF2; the QEA support system can be seen under the nearest quad. The lowest part is a concrete base block, which was evaluated to be the best option for stability and was made in various lengths to match the lengths of all styles of ATF2 magnets. The blocks were fixed to the concrete floor with adhesive polymer concrete and their heights were adjusted by varying the polymer thickness to account for floor height variations. All the ATF2 magnets must be very mechanically stable; this requirement influenced the design of their supports and, anticipating the gradual movement of the supports and magnets caused by thermal variations, all the $\mathrm{FF}$ quadrupoles and sextupoles were put on remotely controlled 3-axis movers, recycled from the FFTB at SLAC, one can be seen in the photo. Each mover has three camshafts, and can adjust horizontal and vertical position and a rotation angle as well, with a position precision of about $1 \mu \mathrm{m}$. Deliberate weekly movements keep the magnets aligned.

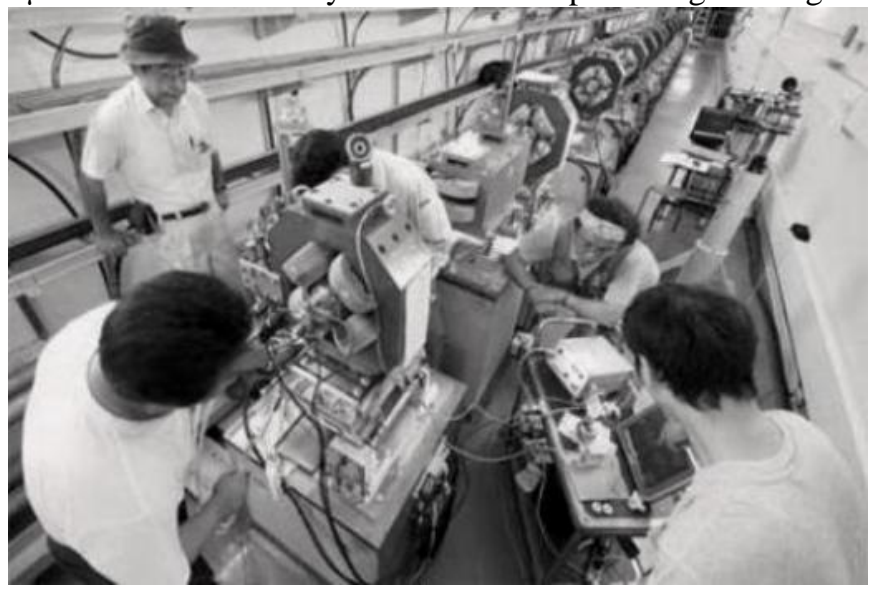

Fig.2 Aligning the QEA quads in the ATF2 final focus section; multiple parts of the QEA support system: custom-made concrete block, mechanical mover and height adjustment plates are visible

\section{B. 3 FF dipoles B1, B2, B5}

The basic requirements for the $3 \mathrm{FF}$ dipoles could not be met with any existing magnets so a brand new dipole was designed; its design and drawings were done at SLAC and 3 dipoles were fabricated and measured at IHEP, Beijing. One of them is shown in the beamline in Fig. 3.

\section{3 FF sextupoles SD4, SF5, SF6}

We used refurbished Stanford Linear Collider FF water- 


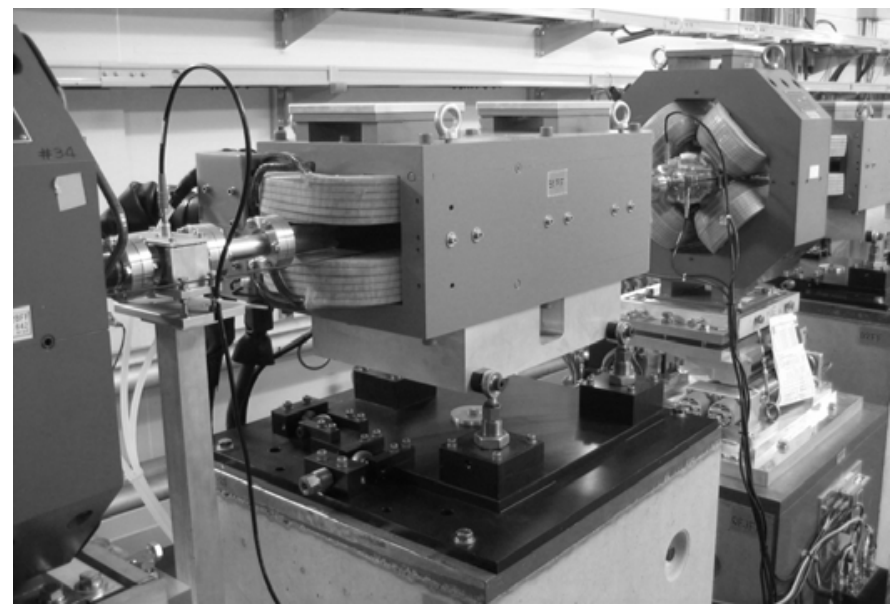

Fig.3 FF dipole in the ATF2 beamline, between 2 QEAs. On manually adjustable support recycled from the Stanford Linear Collider (SLC) at SLAC

cooled sextupoles for these 3 ATF2 sextupoles. All ATF2 magnets that came from SLAC were thoroughly measured by the SLAC Magnetic Measurements Group and Fig. 4 shows one of the FF sextupoles on the rotating coil measuring stand at SLAC.

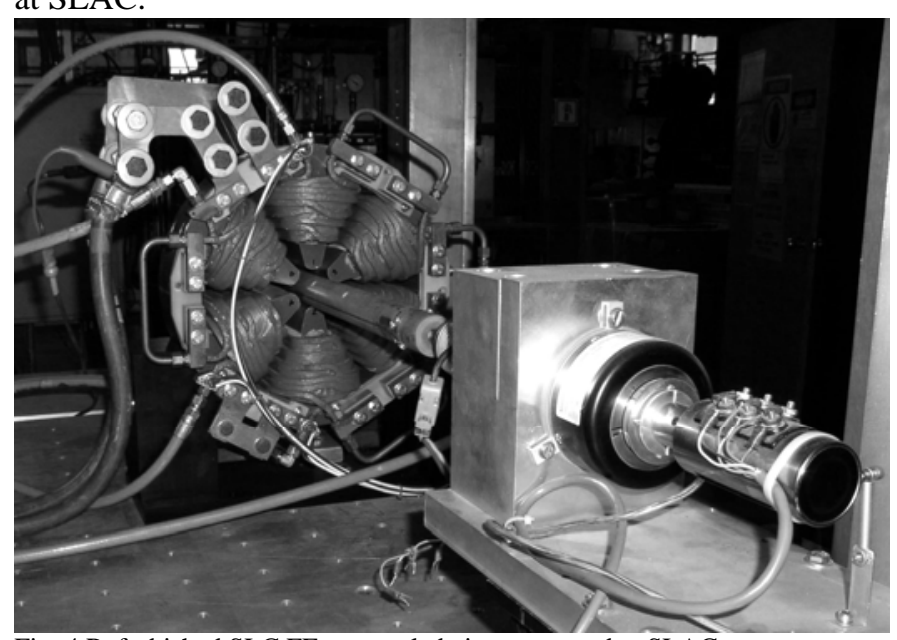

Fig. 4 Refurbished SLC FF sextupole being measured at SLAC

\section{D. "Final Doublet" (FD) quadrupoles and sextupoles}

The four "final doublet" (FD) magnets that do the final focusing of the beam to the nanometer size spot at the interaction point were all modified SLAC magnets from the now dismantled FFTB. The FD sextupoles were solid wire sextupoles, running at 4 - 7 amps. To keep their temperature rise below $\sim 5^{\circ} \mathrm{C}$ we added copper cooling pipes carrying Low Conductivity Water (LCW) and copper plates around the coils.

Some old FFTB quadrupoles, style “QC3”, were modified by placing precisely ground shims in the 4 split planes, to increase the aperture diameter from $35 \mathrm{~mm}$ to $50 \mathrm{~mm}$. We wished to avoid the high cost of machining back the very nice hyperbolic poletips and at their larger radius they created a too high 12 pole. So additional "pole-side" shims were developed to add a small "bump" at each end of the poletips and bolted to the pole sides to minimize the 12 pole component. The need to keep all the multipoles to a minimum put tight mechanical tolerances on the adjacent-pole spacings; all 8 had to be the same to $+/-0.013 \mathrm{~mm}$, and the aperture diameters had to be the same to $+/-0.0254 \mathrm{~mm}$. The modified quads were assembled and measured at SLAC, and a couple of these tolerances were missed, it was not possible to achieve the 4 bore diameters and the 8 gap values simultaneously on both quads. Their measured multipoles were acceptable. The quads were then shipped to LAPP in Annecy-le-Vieux, France where they were integrated into a special support system (see next section) and then they were shipped to KEK, Tsukuba, Japan where they were split in half so a large BPM, designed and made in Korea, could be installed in their bores. The splitting and reassembly process were practiced at SLAC so detailed torqueing instructions could be developed to be followed at KEK, because most spacings could not be reached to be checked after the BPMs were installed. Measurements of the few spacings that could be reached indicate the quads went back together with exactly their original spacing dimensions; this was a great relief after their long journey.

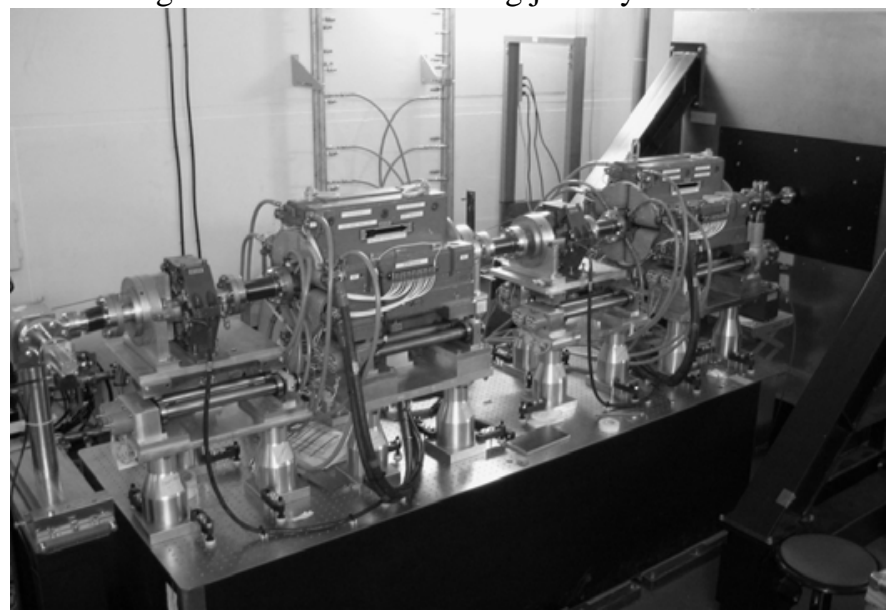

Fig. 5 FD magnets, SF1,QF1,SD0,QD0, sitting on the special support table on their extra support "legs" to arrive at the correct beam height, and with their special BPMs in their bores. Shintake Monitor is wall-like item to the far right

\section{Supporting the Four Final Doublet Magnets}

The last 4 magnets in the ATF2, the FD magnets, have the tightest requirements for their mechanical stability. The FD support table was designed and built at LAPP in France [4]. Here are its requirements (see also Fig 6.):

- Must minimize effects of ground motion

- Must not amplify vibrations in this frequency range : $0.1 \mathrm{~Hz}-100 \mathrm{~Hz}$

- $\quad$ Support that can evolve as FF design evolves

- Control relative motion between FD magnets \& Interaction Point, where Shintake Monitor (SM) sits

- $\quad 7 n m$ vertical jitter tolerance, so beam spot measurement by the SM will have a $<2 \%$ error

- $1.2 \mathrm{~m}$ beam height

There are 2 sources of excitation which can make the FD magnets vibrate: ground motion, whose amplitude depends on time (the coherence does not) and LCW flowing through the FD magnets, this has been designed to be low flow. The quads' relative motion due to cooling water was measured to be much less than $0.1 \mathrm{~nm}$, this is excellently small.

Various methods of attaching a honeycomb (vibrationdamping) table to the floor were tried and the vibrations of the magnets were measured in the $\mathrm{X}, \mathrm{Y} \& \mathrm{Z}$ directions from $0.1 \mathrm{~Hz}$ to $100 \mathrm{~Hz}$ with 3 models of vibration sensors, the Guralp CMG-40T [5], Endevco 86 [6] \& TOKKYO KIKI 


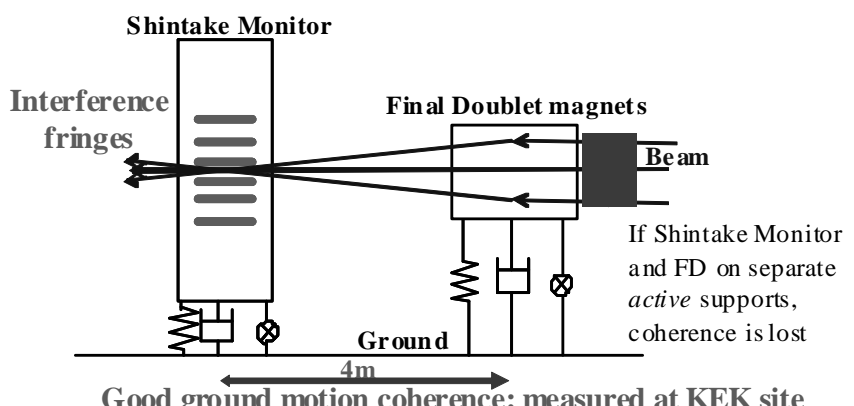

Good ground motion coherence: measured at KEK site

Fig. 6 Explanation of approach chosen for FD support table

MRA-06X [7]. The optimum set-up was found to be to tie the table block, without legs/feet, to the floor with steel plates and put a thin layer of natural beeswax [8] between the block and the plates. Ground motion was measured extensively too. The tightest tolerance on any beam component motion is on the relative vertical motion, above $0.1 \mathrm{~Hz}$, between the Shintake Monitor (SM) and QD0; it must be less than $7 \mathrm{~nm}$. This relative motion, and motion perpendicular and parallel to the beam, were measured in the worst case, when the absolute ground motion had its highest observed amplitude during a 72 hour period. The integrated Root Mean Squares (RMS) of the 3 motions are shown in Fig 7. Various transfer functions are used to generate Fig 7. and 1\% measurement errors in one function are amplified by two huge peaks of floor motion at $3.5 \mathrm{~Hz}$ and between 0.2 and $0.4 \mathrm{~Hz}$ (a global tidal effect), leading to the apparent increases in integrated RMS at those frequencies. In fact the coherence and phase transfer functions below $4 \mathrm{~Hz}$ are almost at 1 and so ground motion should not increase relative motion below this frequency. So we use $5.1 \mathrm{~nm}$ as the measured vertical relative motion for SM-QD0.

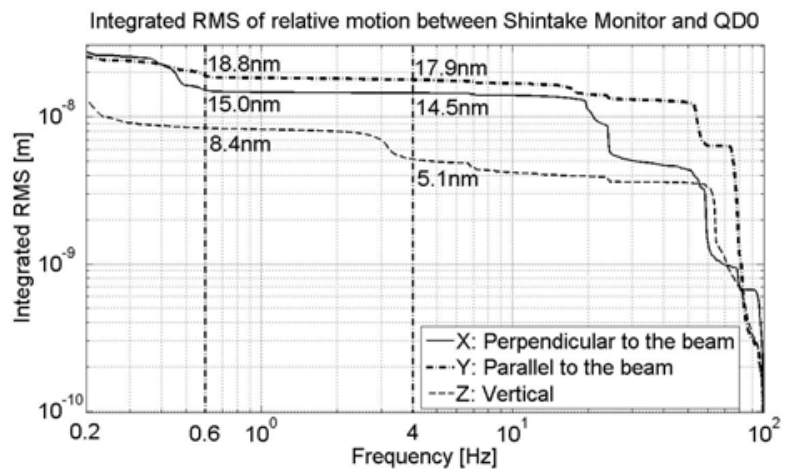

Fig. 7 Integrated RMS of relative motion between Shintake Monitor and QD0

Measurements of the FD support system showed it met all vibrational (=relative motion) tolerances, see data in table II.

TABLE II. SUMMARY OF VIBRATION MEASUREMENTS

\begin{tabular}{lcccl}
\hline \hline Dir & $\begin{array}{c}\text { Tolerance } \\
(\mathbf{n m})\end{array}$ & $\begin{array}{c}\text { Meas. QD0 } \\
\text { to SM (nm) }\end{array}$ & $\begin{array}{c}\text { Meas. QF1 to } \\
\text { SM (nm) }\end{array}$ & $\begin{array}{l}\text { Absolute } \\
\text { Motion } \\
(\mathbf{n m})\end{array}$ \\
$\mathbf{X}$ & $\sim 500$ & 14.5 & 16.6 & 578.8 \\
$\mathbf{Y}$ & $\sim 10000$ & 17.9 & 16.5 & 548.5 \\
$\mathbf{Z}$ & $7(\mathrm{QD0})$ & 5.1 & 6.5 & 212.6 \\
& $20(\mathrm{QF} 1)$ & & & \\
\hline \hline
\end{tabular}

\section{Alignment of the ATF2 MAgnets}

The first alignment of the FF magnets was started in August 2008. A laser tracker SMART310 of Leica with a mirror target was used to observe the horizontal position, a N3 leveling telescope of Leica with an optical target to observe the height and an electric level Talyvel-4 made by Taylor-Hobson to observe the levelness. See Fig. 2 for a photo of this process.

A second alignment, a smoothing process, was performed at the end of September 2008 [9]. After correcting the positions of several magnets, we finally obtained the alignment results shown in Fig. 8. The plot shows satisfactory deviations of each magnet's height from a fitted smooth curve, with a total RMS deviation of $80 \mu \mathrm{m}$. "KP" points are alignment monuments that were not used in this process.

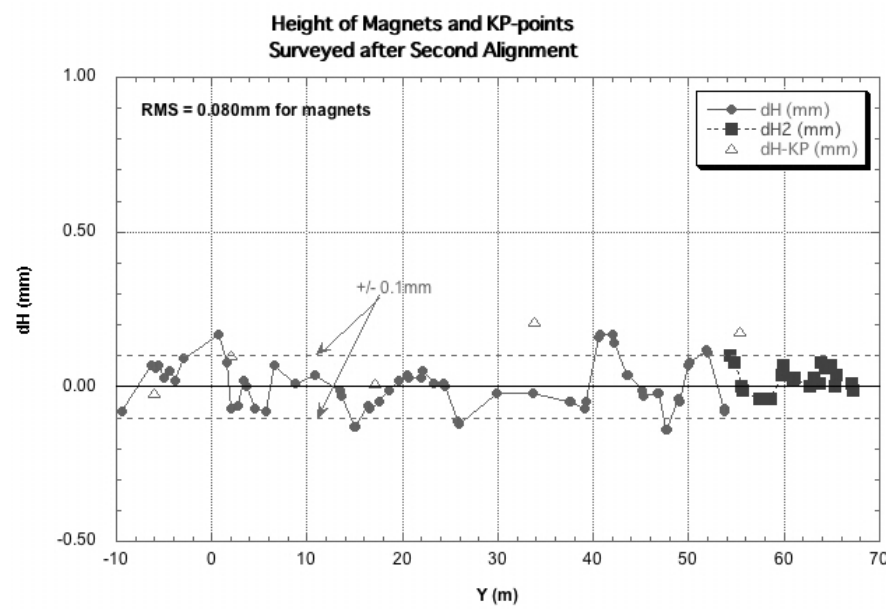

Fig.8 Deviations of height of ATF2 FF magnets from a fitted smooth curve

\section{CONCLUSIONS}

All 5 "new" magnet styles provide the integrated fields as required within the current and voltage limits of the new power supplies. Except for the QEAs the new styles' field qualities meet stringent requirements. The QEA's with higher multipoles were assigned to positions with looser tolerances. [3]. All operate with low temperature rises in their LCW $(\sim<$ $3^{\circ} \mathrm{C}$ ) which minimizes thermal effects and the specially designed magnet supports keep vibrational movements below tolerances. The ATF2 beamline is operating now; it is progressing towards the $37 \mathrm{~nm}$ vertical beam spot size goal.

\section{REFERENCES}

[1] ATF2 Proposal. SLAC Report 771. August 2005.

[2] T. Shintake, "Proposal of a Nanometer Beam Size Monitor for e+eLinear Colliders”, Nucl. Instr. Meth. A311, pp453-456 (1992).

[3] M. Masuzawa and R. Sugahara, "Field Measurements of the ATF2 Quadrupole Magnets Manufactured by IHEP”, IEEE Trans. Appl. Supercon., to be published.

[4] B. Bolzon et al, "Linear Collider Final Doublet Considerations: ATF2 Vibration Measurements”, Proceedings of the Particle Accelerator Conference 2009, http://accelconf.web.cern.ch/accelconf/p09/PAPERS/th5rfp087.pdf

[5] Guralp Company http://www.guralp.com/products/40T/.

[6] Endevco Company http://www.endevco.com/product/Product.aspx?product id=105 .

[7] Tokkyo KiKi http://www.tokkyokiki.co.jp/english/3.html .

[8] Beeswax from Ickowicz http://www.ickowicz-apiculture.com/.

[9] R.Sugahara, "Alignment Status of the ATF2 Magnets," unpublished report to an ATF2 Collaboration Meeting, KEK, September 2009. 